\title{
Bottleneck-oriented Logistic Analysis as a Basis for Business Re-engineering
}

\author{
T. Penz, P. Nyhuis \\ Institute for Production Systems, University of Hannover \\ Callinstraße 36, 30167 Hannover, Germany \\ Tel: $\quad+49-511-7623494$ \\ Fax: $\quad+49-511-7623814$ \\ E-mail:pz@thor.ifa.uni-hannover.de
}

\begin{abstract}
To ensure short delivery time and high delivery performance it is necessary to have control over manufacturing procedures and to keep lead times to a minimum. At the same time production profitability must be guaranteed. The interaction between these (and other) objectives and occurring conflicts can be investigated with bottleneck-oriented logistic analysis, which makes it possible, for example, to determine ideal states and realizable values for lead times and inventory for existing or planned production structures. In addition, the system behaviour of the production can be investigated with varied side constraints; also the effects of measures taken to influence the logistics potential can be described qualitatively and quantitatively. This allows an objective-oriented optimization of the manufacturing procedures and structures with regard to business re-engineering.
\end{abstract}

\section{Keywords}

Bottleneck-oriented logistic analysis, logistic operating curves, logistics positioning, simulation.

\section{INTRODUCTION}

The strategic importance of production logistics will undoubtedly continue to increase in the future. Particulary under conditions where far-reaching changes in progressively shorter periods are required, they have significantly influenced the fulfilment of market demands and company objectives. Regarding the concept of maximizing the efficiency of the production sequence, production logistics should cater for the realisation of such objectives as maximizing schedule performance and loading, as well as minimizing lead time and inventory.

Employing only the currently available logistic systems and instruments in industrial companies is not enough. Rather, it must be guaranteed that the existing interdependence between the logistic objectives in these systems is adequately considered, in order to facilitate a targetoriented structuring and control of material and production flows. To this effect, general logistic models are required to describe the dynamic correlations between the afore-mentioned logistic quality features. Furthermore, the actual and the scheduled production flow of orders should be realistically described. 
The funnel model and the throughput diagram represents such a general model (Figure 1). Throughput diagrams enable a qualitative precise description of the dynamic behaviour of work centres. Furthermore, the four primary quality features of production (schedule performance, inventory, lead time and loading) can be depicted and a numerical calculation of the corresponding determinants can be effected (Wiendahl 1987). In this way, the pertinent relationships between the logistic objectives can be demonstrated and a mathematical description derived therefrom.

However, research has shown that evaluation based solely on the graphs and key data obtained from simulated as well as real production sequences is often inadequate to fully describe the dynamic behaviour of production systems, especially the relevant correlations between the logistic objectives. For this reason it has been found useful to condense the results of various stationary states into what are termed "logistic operating curves". These allow an identification of the dependence of diverse objectives on the changes in parameters. In the meantime, it has also been proved that a target-oriented evaluation and optimization of process flows and structures is possible using such logistic operating curves.

Logistic operating curves facilitate the representation of the logistic determinants of performance, lead time and range as a function of inventory (Figure 1 at the bottom). It can be seen that the performance changes only negligibly above a specific value of inventory. At this point, there is always enough work in process so that work interruptions as a result of obstructions in material flow do not arise. Below this inventory value, however, increasing performance losses occur as a result of insufficient work supply.

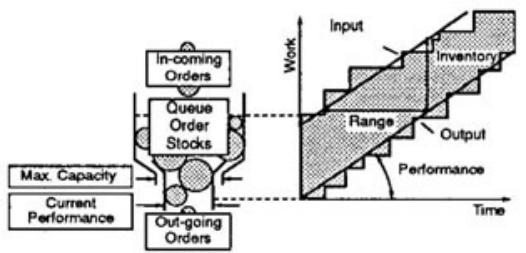

a) Funnel Model and Throughput Diagram
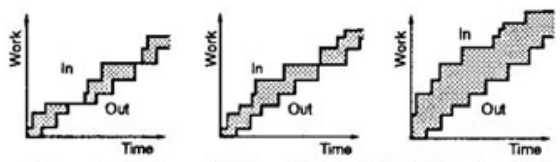

I: Lower Load Limk II: Optimum Operation Point IIt: Upper Load Lime

b) Typical Production Situation

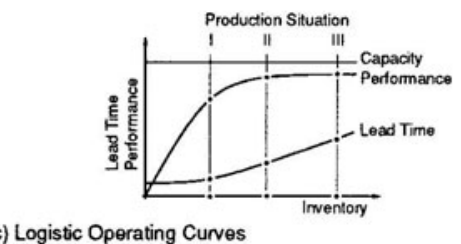

(c) IFA C0476E

Figure 1 From the Funnel Model to the Logistic Operating Curves. 
The lead time respective range in contrast increases proportionally with the inventory above a specific inventory value. A reduction in the inventory leads to a decrease in the lead time. However, the minimum value of the lead time cannot fall below a certain minimum, determined from the operation time of the orders and the transit time, as the case may be. With the aid of the operating curves the interdependencies between the logistic factors can be shown for any work centre. They illustrate the dilemma of the forward scheduling, revealing the mutual enhancement between some of the logistic objectives, as well as a reciprocal interference in others. As a result, there is no single objective whose value could be maximized or minimized; rather, the effects of measures respecting all the elements must be considered simultaneously.

\section{POTENTIAL APPLICATION POSSIBILITIES OF LOGISTIC OPERATING CURVES}

The logistic operating curves identify the determined system behaviour of a production corresponding to the quality features when the most important adjustable parameter of the production control changes: this parameter is the inventory. Additionally, it is possible to represent different operating curves with varying production and order structures. These can be compared with each other, thereby providing a means of assessing the effects of measures carried out in the production flow under logistic aspects. Figure 2 shows in a summarised overview that, using this technique, new possibilities for quantifying logistic rationalization potentials are presented.

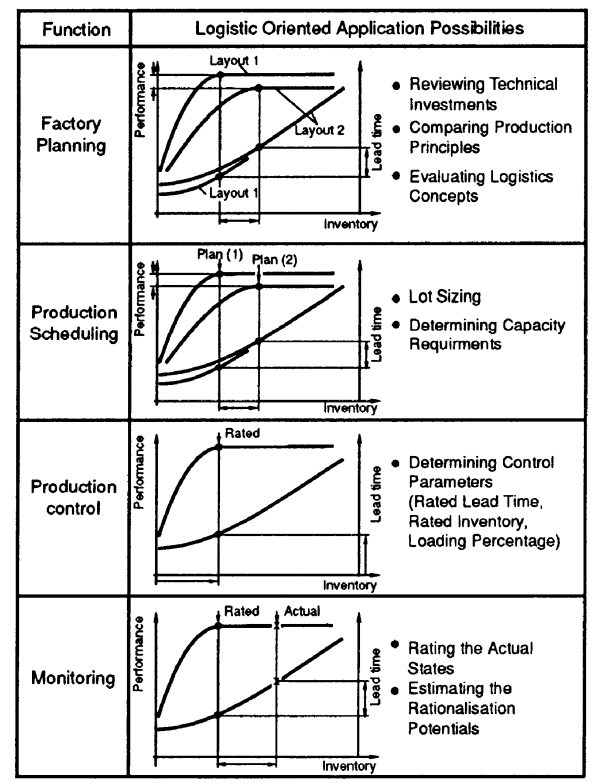

() IFA C0735E

Figure 2 Possible Application for Logistic Operating Curves. 
In this way, operating curves can be employed to evaluate the process flows within the scope of a monitoring. It can be shown which lead times and inventories could have been achieved with the existing structural constraints without significant bottlenecks in the material flow and accompanying performance losses.

Besides, a differentiated determination of the rated lead times and other control parameters for the production control is possible. Representing the quality features in a diagram enables a decision to be made as regards the most important feature, depending on the current production and/or market situation, as well as the constraints specific to the work system. The relevant parameter adjustments which should be made to the PPC system can also be decided upon at any time. At the same time, it can be shown which consequences can be expected with regard to the logistic quality features with a change in parameters.

The afore-mentioned areas of application aim at indicating the existing logistic potentials, analysing deficits, or sustaining the logistic state. Should it turn out, within the scope of these applications, that objectives cannot be realised without supporting measures, the operating curves can also be enlisted for a logistic evaluation of planning alternatives. Investment decisions (deployment of new transport systems, introduction of new production technologies,capacity expansions, ...), the choice of an alternative lot-sizing process or measures in the field of business re-engineering (design of manufacturing islands, outsourcing, ...) can be reviewed with regard to their importance for the realisable lead times, inventories and loads.

\section{METHODS OF DETERMINING LOGISTIC OPERATING CURVES}

Before introducing the different types of operating curves and their forms of determination it should again be pointed out that operating curves are used to describe possible diverse production states. A momentary state in the production always corresponds to a single operating point on a curve. The curve itself shows how the pertinent production or work centre would have behaved if a different inventory level had been set, the other constraints remaining constant.

This clarification indicates that, using analysed data of process chains recorded at different instances, real logistic operating curves can be reconstructed. This assumes a significant change in the inventory within the evaluation period, resulting in different operation points, with other constraints remaining constant. This refers essentially to the structure of the capacities, and the orders to be handled. Since the necessary evaluation time is normally very long, thereby making a fulfilment of the above-mentioned demands very difficult, real operating curves for practical purposes will seldom find application.

A second possibility is the simulated operating curve. Simulation systems provide the means of reproducing a real system using computer programs. In this way, the behaviour of the system with a specific change in the simulation conditions can be described, analysed and optimized. In the past, several researchers have presented simulation methods which describe the relationships between the different production objectives, in the afore-mentioned fields of application (e.g. Rice/Gross, 1990; Kuprat, 1991; Kuhn, 1992; Zäpfel, 1992).

The third type of curve is the ideal logistic operating curve. Based on an analytical consideration of ideal production flows and the derived ideal process key data, ideal production flows were modelled (Nyhuis, 1991). By this means, an order is handled directly on its arrival at the work centre. Hence there is no interoperation time between two operations. Under this restriction, the inventory is determined directly from the work content of the orders to be processed. This depicts the lowest limit of the inventory, which cannot be gone below even under ideal conditions, without causing inevitable loading losses caused by the inventory. Limits and ideal operating curves for the lead time and the range can be established (Nyhuis, 1993). 
In the meantime, analyses of several simulation results has made the derivation of an approximation equation possible, enabling a mathematical determination of the operating curves. The approximation equation for these theoretical curves as well as the corresponding parameters and the application constraints are fully described in (Nyhuis, 1993). In order to give a rough sketch of the application possibilities of theoretical curves the parameters of the approximation equation are explained in the following.

The two most important parameters of the equation are the "maximum possible performance" and the "ideal minimum inventory". These two factors, which also determine the ideal operating curve, are derived from work-centre-specific or production-order describing determinants, which are normally present in every company.

Consequently, the 'maximum possible performance' variable is derived from the capacity. The upper performance limit of a work centre is fundamentally determined from the restricted capacity factor (production facilities or personnel). Additionally, it must be observed that, owing to capacity-reducing disruptions in production (e.g. machine breakdowns), the maximum possible loading can be limited further. Furthermore, as long as the capacity is given in loading hours rather than in standard hours, this must be corrected with the performance rate when determining the maximal possible performance.

As illustrated above, the ideal minimum inventory describes the value needed under ideal conditions to make use of the maximal possible performance. It is determined essentially from the work content of the orders: the minimum inventory depends particularly on the variance of the order times besides the mean value. Via the order times, the determination of the operating curve is also affected by the lot size, setup times and the processing times. In addition, it can if necessary be taken into account that an order in stock could be related to the minimum interoperation times because of transportation, or other factors (e.g. cooling times of hot parts).

Furthermore, via structure parameters, the coupling of the relevant work centre to the production flow can be described. In this way, it can be taken into account whether each work centre possesses its own inventory buffer or whether a common buffer exists for several work centres. In the second case, the risk of an interruption in the material flow for a particular work station is reduced, so that the necessary aggregate buffer can be reduced.

Should the parameters in Figure 3 be present, the operating curve can be directly determined with the said approximation function. A change in any of the parameters results inevitably in a different curve. Consequently, the approximation equation can be employed to investigate and assess the effects of different influencing measures on the logistic potential of a work centre, qualitatively and quantitatively, and if need be to establish a basis for comparisons.

\section{APPLICATIONS}

The application possibilities of determined logistic operating curves are manifold. It was illustrated in Figure 2 that these curves can be employed to predict the qualitative and quantitative effects on the logistic objectives.

The following examples stem from an industrial project which was carried out jointly with a manufacturer of measuring instruments. The primary goal was to investigate the application possibilities of determined operating curves as supplement to a commercial monitoring system.

The particular task here was to examine whether the real process flows in the department of "insertion of printed-board" could be represented well enough with the approximation equation. After confirming this, and based on the determined operating curve, the existing logistic potentials and the measures aimed at by the company for reducing the lead time and inventory were discussed and analysed. In a subsequent investigation, it was examined whether the 
predicted improvements in the production flow could be implemented.

In the first stage of the project, a comprehensive analysis of the lead time and the inventory was carried out. The results are shown in a highly condensed form in Figure 3. With the aid of this graph, which depicts both the logistic operating curve and the vital logistic data, the most critical work centre as regards order flow (i.e. giving rise to lead time) can be identified. Furthermore, using the logistic operating curves, it is possible to determine the work centres at which a reduction in the lead time could be effected, using control measures alone.

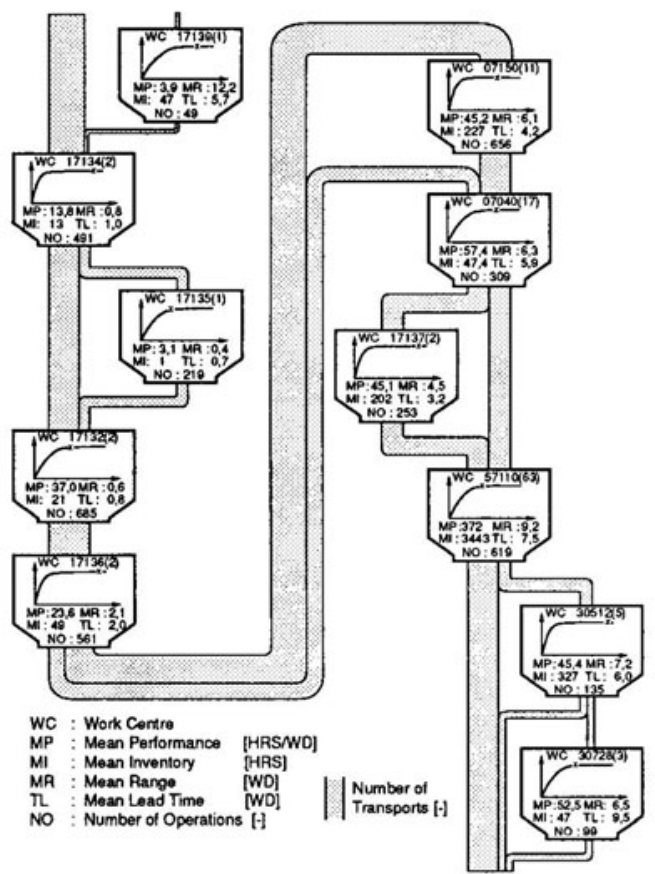

(c) IFA 7671E

Figure 3 Logistic Score Data in the Material Flow (Extract).

The measured operation point of these systems is located well to the right of the transitional range. In contrast, for work centres where the operation point is located in the transitional region, it is necessary to effect structural changes (orders, capacities, layout,...) in addition to the control measures, if significant reductions in the lead time are to be realised. Two examples from the investigation in which different procedures could be derived will be discussed in the following.

In the first work centre the concern was with 'SMD insertion automats' (WC 17137) (two single work stations) which were fully loaded on a three-shift operation basis. The throughput diagram of this work centre for an investigation period of 6 weeks is shown in Figure 4. The trends of the work input, output and inventory are shown as a function of time. Additionally, the lead time elements have been entered, which describe the individual lead times of the different orders through the system. 


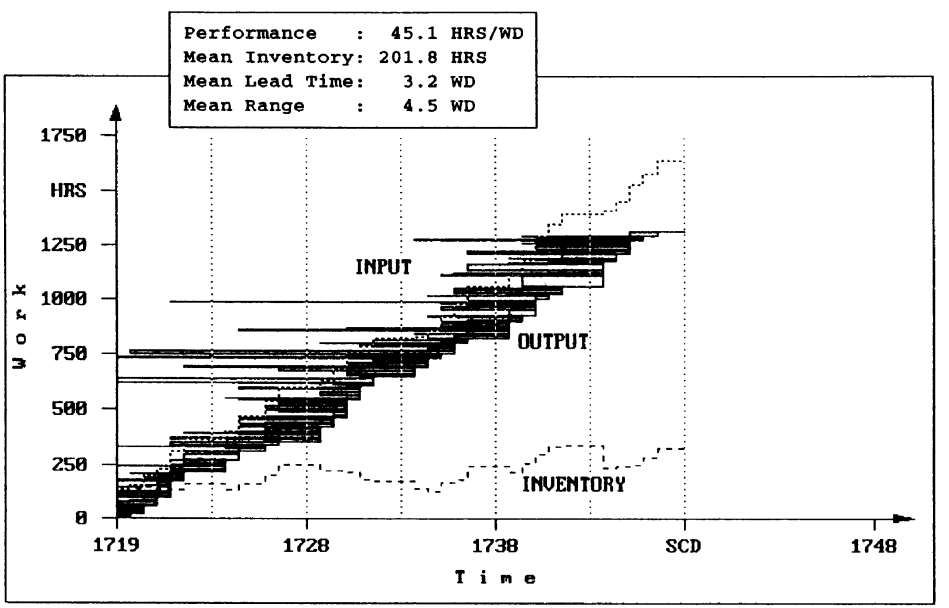

(-) IFA C2413E

Figure 4 Graphic Results of the first Analyses for the 'SMD Insertion Automats'.

It can be observed from the diagram that fluctuating inventory was present at the work centre during the evaluation period. The work input was obviously not tuned to the output or the capacity of the work centre. Moreover, the lead time elements show that many changes in order sequence were carried out. The existence of changes in order sequence indicates that inventory reductions are possible, for these changes in sequence can only be carried out, if several orders are simultaneously present in the queue.

On the whole, the results show that the existing mean inventory of about 200 hours is clearly higher than the level necessary to maintain performance. In order to quantify this, the operating curves for the subsequent analysis were determined from the minimum inventory (resulting from the order times of the completed orders, see above), the ratio of current inventory to minimum inventory as well as the realised mean performance, with the aid of the approximation function. These curves as well as the actual values of performance, range, lead time, and inventory are shown in Figure 5.

In addition, 'appropriate value range' of the key data is indicated. The limits of this range were set at 1.5 to 2.5 times the ideal inventory in agreement with the company. The graph shows firstly that the measured operating state lies clearly in the over-load region. It can be seen that it is possible to reduce the inventory and the lead time significantly merely by instituting appropriate inventory control. Even presuming that high loading must be ensured at this work centre, it can be assumed that an mean inventory of about 60 hours is sufficient to guarantee the necessary performance. This would result in a reduction of the lead time of 3.5 work days to 1.5 work days. The restructuring measures planned by the company for increasing the insertion rate could hence be shelved, since at the time in question no advantages of any consequence could be expected from them from the logistic standpoint. For this reason an investment of tens of thousands German marks could be saved.

The subsequent analysis carried out after nine months (over a period of six weeks as well) confirmed the previously established assertion with respect to the existing rationalisation 
potential. The analysis showed that the inventory for this work centre could be reduced, on an average, to 94 hours (by almost 55\%). In the case of a higher daily performance (47.6 instead of 45.1 hours per work day), the range and the lead time are reduced from 4.5 to 2.0 work days (WD) and from 3.2 to $1.9 \mathrm{WD}$ respectively.

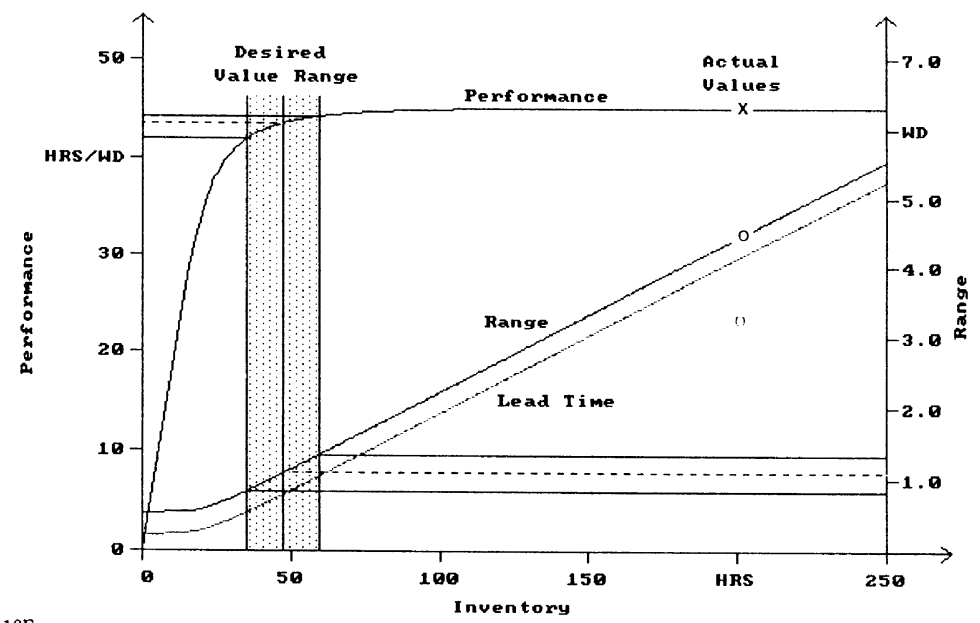

๑ IFA C2418E

Figure 5 Determined Operating Curves for the 'SMD Insertion Automats'; 1. Analysis.

The example discussed above shows that it is possible to realise considerable reductions in the lead time and the inventory, without altering the structural constraints. Within the scope of the conducted analysis, there were also a number of work centres where it could be proved that, in order to increase the logistic potential, changes in the structure of order time or in the capacity situation were necessary.

An example of such a work centre is the 'Segment Production' (WC 57110) (manual insertion of component with 63 work stations of which an average of 48 were in one-shift operation). Figure 6 illustrates the production flow measured in the first investigation. The diagram shows that a fairly uniform inventory was present at the work centre on the whole. The input is fairly well geared to the output. But at the same time the numerical evaluation of the feedback data shows that the range is $9.2 \mathrm{WD}$ and the mean lead time 7.5 WD.

Since these lead time values were clearly higher than all those obtained from the other analysed work centres, and the 'segment production' represented the most important capacity of the production department, the cardinal importance of this work centre from the logistic viewpoint is accounted for. The operating curves showed that these extremely high values of the lead times can still be considered as reasonable, under the prevailing conditions. Reducing the inventory and the lead time solely through production control measures would not have been possible for this work centre, if the company had not accepted performance losses at the same time.

While discussing the analysis results, it came to light that the required high level of lead times and inventories was attributable in particular to the existing structure of the order time. The analysis of the order times showed that the distribution of the order times was marked by 
wide mean variation, as well as high mean value. However, since the function for determining the ideal minimum inventory is quadratic with respect to the standard deviation (Nyhuis, 1991), it could be concluded that orders with a high work content should be reduced specifically to achieve a harmonization of the order structure. Accordingly, after the analysis, the company subdivided all orders with order times higher than 50 hours and pooled requirements. Furthermore, it was decided that the orders with order times higher than 30 hours should be handled by two or more workers in parallel. These two measures, affecting roughly $18 \%$ of the operations and about $55 \%$ of the total work content, led to a reduction of the ideal inventory by $35 \%$.

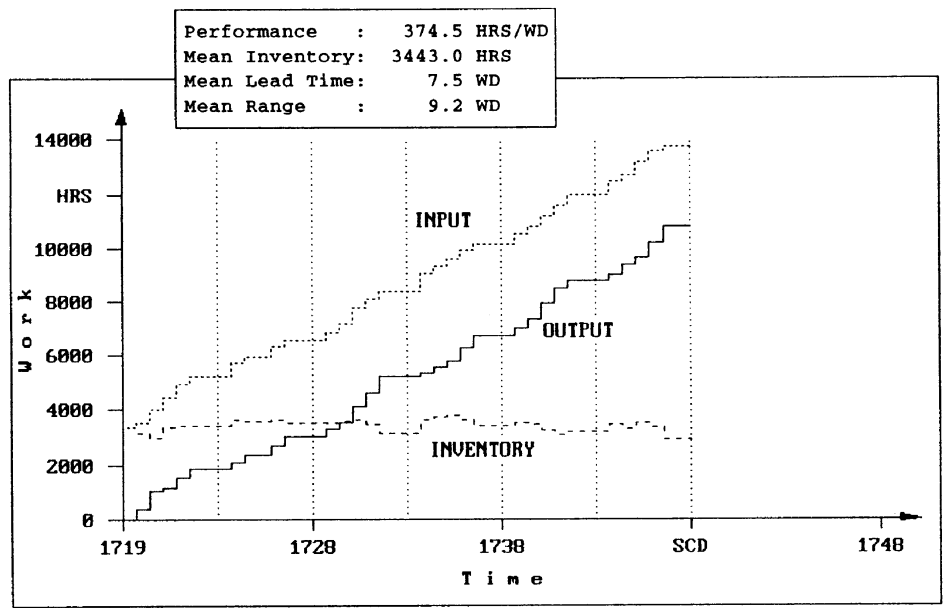

(c) IFA C2416E

Figure 6 Graphic Results of the first Analyses for the Work Centre 'Segment Production'.

The success of these measures was reflected in the process flow measured during the subsequent analysis and in comparison between the operating curves and the logistic data for the two analyses (Figure 7). Inventories and lead times could be reduced by more than $30 \%$ without incurring further performance losses.

However, it must be stressed here that is was not alone the measures aimed at harmonizing the order times that brought this reduction. Rather, the company also succeeded in utilizing the ensuing potentials by reducing the inventory to the targeted level through production control measures

\section{SUMMARY}

Using the operating curves, the interdependencies between the logistic objectives of lead time, inventory and performance can be described as a function of the company-specific constraints. Hence, they lend themselves as tools for demonstrating the effects of measures aimed at influencing these objectives, and consequently support corporate decisions, particularly in the area of production planning and control (PPC) and factory planning. 


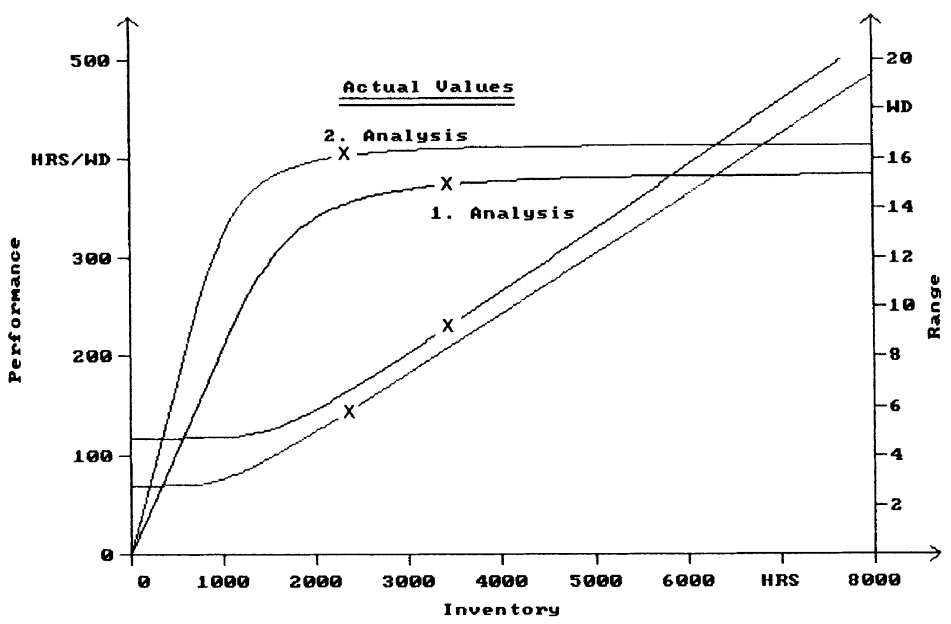

(c) IFA C2414E

Figure 7 Comparison the Determined Operating Curves for both Analyses; Work Centre 'Segment Production'

Hitherto, the generation of such logistic operating curves was only possible via simulation. The mathematical model for determining the operating curves enabled a satisfactory projection of real process flows in production despite the at first sight considerable simplifications. The pioneer prototype applications of determined curves with monitor systems show that with this model differentiated predictions respecting the attainable lead times and the inventories can be derived.

\section{REFERENCES}

Bechte, W. (1984) Steuerung der Durchlaufzeit durch belastungsorientierte Auftragsfreigabe bei Werkstattfertigung. Fortschritt-Berichte VDI, Row 2, No. 70, Düsseldorf.

Kuhn, A. (1992) Modellgestützte Logistik - Methodik einer permanenten ganzheitlichen Systemgestaltung, in Rechnergestützte Fabrikplanung '92. VDI-Berichte 949, Düsseldorf.

Kuprat, Th. (1991) Simulationsgestützte Beurteilung der logistischen Qualität von Produktionsstrukturen. Fortschritt-Berichte VDI, Row 2, No. 228, Düsseldorf.

Nyhuis, P. (1991) Durchlauforientierte Losgrößenbestimmung. Fortschritt-Berichte VDI, Row 2, No. 225, Düsseldorf.

Nyhuis, P. (1993) Betriebskennlinien zur Entscheidungsunterstützung in der Produktionsplanung und -steuerung, in Neue Wege der PPS (ed. H.-P. Wiendahl), gfmt-Verlag, München.

Rice, W. und Gross, R. (1990) Simulation Technique and the WIPAC Curve for Balancing Capacity against Inventory. APICS, 33rd International Conference Proceedings, New Orleans.

Wiendahl, H.-P. (1987) Belastungsorientierte Fertigungssteuerung. Grundlagen, Verfahrensaufbau, Realisierung. Hanser-Verlag, München, Wien.

Zäpfel, G. and Hödlmoser, P. (1992) Läßt sich das KANBAN-Konzept bei einer Variantenfertigung wirtschaftlich einsetzen? Zeitschrift für Betriebswirtschaft, 62nd Annual, No. 4. 